\title{
Possibility of species extinction in the periodical forced prey-predator model
}

\author{
Aldona Krupska \\ Institute of Molecular Physics PAS, M. Smoluchowskiego 17, 60-179 Poznań, Poland. \\ Accepted 17 April, 2013
}

\begin{abstract}
The response of the Lotka-Volterra system forced by periodical modulation was numerically simulated in a wide frequency range. The main question is: how does the change of environment $A$ parameter influence the behavior of such a system? The answer to this question is the main purpose of this paper. In the resonance region, the oscillation of the system fits to the forced frequency (or its harmonics) and shows that the numbers of prey and predators change significantly in time. Their populations after reaching the specified value become small and sometimes they are near zero and making the recovery of the population (e.g. of the predators) impossible. The Lotka-Volterra non-linear system, in contrast to the linear oscillatory systems (e.g. damping harmonic oscillator- RLC circuit), is very sensitive; the initial conditions of the effect of resonance chaos depend on the conditions of the system. Superposition principle, including relaxation behavior is not applicable in the nonlinear Lotka-Volterra system.
\end{abstract}

Key words: Population model, oscillatory inflow, nonlinearity, resonance, higher harmonics, species extinction.

\section{INTRODUCTION}

In spite of many studies that are devoted to the relation between prey and predator in the evolution dynamic (in particular, the species extinction), there is lack of a satisfactory model of that ecosystem. The simple model is based on Lotka-Volterra ecological system and it has been considered from many points of view (Nicolis and Prigogine, 1977; Smith, 1978). There are also more specific modifications of the Lotka-Volterra model: Rosenzweig and McArthur model (Rosenzweig and MacArthur, 1969; MacArthur et al., 1966), SI model (Svennungsen and Kisdi, 2009; Gilchrist and Coombs, 2006) and its combinations (Morozov and Adamson, 2011) as well as Arditi and Ginzberg (1989) modifications.

The oscillatory aspects in the Lotka-Volterra system are presented by many authors. The exogenous component a strong periodic component, called periodic forcing is regarded by Turchin in his book (Turchin, 2003). The author shows how nonlinear population dynamics can create something novel when interacting with an exogenous driver. He demonstrates how two regular, periodic kind of motions produce an irregular trajectory. The Lotka-Volterra dynamics under periodic influence is considered by Dutta and Bhattacharjee (2008). Some aspects of evolution as well as extinction of species based on the Lotka-Volterra model are included in the literature. Evolutionary stability in Lotka-Volterra systems was considered by Cressman and Garay (2003). Extinction dynamics of a Lotka-Volterra model of twolevel food web were considered too (Coppex et al., 2004). Khaminskii et al. (2003) show how perturbations of coefficients averaging lead to extinction of one of the populations. Stabilization of the Lotka-Volterra food webs by evolutionary feedback is presented by Ackland and Gallagher (2004). The dynamic behavior of the classical periodic Lotka-Volterra competing system with impulsive effect is investigated in the paper by Liu and Chen (2004). The authors explain how one of the competing 
species without impulsive effect but in a periodic environment would be doomed to extinction.

The Lotka-Volterra model describes the periodic oscillation in certain autocatalytic reactions and it is used to explain the some biological population behaviors. This model was employed to explain ecological relationships in prey-predator populations (e.g. hares and lynxes). Lotka in 1925 (Lotka 1925) and Volterra in 1926 (Volterra 1926) independently formed nonlinear equations describing prey-predator time behaviors:

$$
\frac{d X}{d t}=a X-b X Y, \frac{d Y}{d t}=c X Y-e Y
$$

Where, $X$ is the number of prey and $Y$ is the number of predators. Parameters $a, b, c$, and $e$ represent the interaction of two species; in particular, a denotes a prey's increasing rate in the absence of predators and $e$ is the predator's mortality rate. The model shows that the prey and predators populations exhibit nonlinear stationary oscillations and their phase trajectory (mutual dependence between $X$ and $Y$ ) is a closed cycle line.

In most cases, a constant inflow of food consumed by prey in the predator-prey system was considered and therefore in equations a constant parameter, $a$ is assumed (1). However, in some real systems, food inflow changes in time. How does the change of a parameter influence the behavior of such a system? The answer to this question is the main purpose of this paper. We have shown through computer simulations the existence of resonance and chaotic effects which result from oscillatory modulation of food inflow to the predator-prey ecosystem. The resonance effect should be observed when a frequency of food inflow into a population area becomes equal to the natural frequency of predator-prey system.

In addition, chaotic effects are particularly visible when the system is far from equilibrium. The resonance and chaotic effects were observed in other nonlinear systems, e.g. for a forced nonlinear pendulum (Kahn and Zarmi, 2003; Arinstein and Gitterman, 2008). The present simulation, when applied to the ecological system, allows for a better understanding of the nature of all nonlinear oscillatory systems in biology.

\section{Computer simulation and results}

For convenience and transparency of calculations, we used dimensionless variables, in which the differential equations (1) look as follows:

$$
\frac{d x}{d \tau}=A x(1-y), \quad \frac{d y}{d \tau}=y(x-1)
$$

Where, $x$ and $y$ denote the prey and predator reduced population, respectively, in stationary conditions:
$x=X \frac{c}{e}$ and $y=Y \frac{b}{a} \cdot \tau=e \cdot t$ is the reduced time, and $A=\frac{a}{e}$ is a constant directly proportional to the resources of food consumed by prey and inversely proportional to the mortality rate of predators. In this form a non-zero stationary state $\left(\frac{d x}{d t}=0 ; \frac{d y}{d t}=0\right)$ is observed for $x=1$ and $y=1$, and populations of prey and predators are constant.

When the initial values of $x$ or/and $y$ are not one, simple analyses reveal steady oscillations of both $x$ and $y$. When the predator population is low, the prey population increases or vice versa. A phase trajectory (called phase portrait or limit cycle), that is, the dependence between $x$ and $y$ is determined. The population oscillation of predators shifts in comparison to the prey population. For the small deviations from the equilibrium, the phase trajectory is almost rounding whereas for the large deviation it becomes similar to triangle in shape. This shift is still large near the equilibrium. This is widely described in literature (Nicolis and Prigogine 1977; Smith 1978). Figure 1 shows the period $T$ of natural oscillations of the system in function of its amplitude, obtained by numerical calculations for the different initial values of $x$ and $y$. For the small deviations from the equilibrium, the beginning of the plot in Figure 1 can easily be calculated by linearization of equations (2).

In this case, we deal with a simple harmonic motion. The population of the predator delays from that one of the prey by $\pi / 2$, and its oscillation period is equal to $2 \pi$. For the larger deviation, the linearization may not be applied and the monotonous rise of the period vs. amplitude is visible. In a similar experiment (Kahn and Zarmi, 2003). This relationship will be further taken into account to interpret the unusual behavior of Lotka-Volterra system in resonance.

In this paper, we assume that the relation between prey and predators, represented by $A$, is not constant but periodically modulated in time. For the sinusoidal modulation, $A$ may be expressed by:

$$
A=A_{0}(1+m \sin (\omega \tau))
$$

Where, $\omega$ denotes the modulation frequency ( $\omega=2 \pi / T$ ) and $m$ is the amplitude modulation depth. $A_{0}$ represents a constant concentration of food and determines the amplitude of the modulation, provided the rate of death of predator is constant. In order to solve differential equations (2) the fourth-order Runge-Kutta numerical algorithm was used (Appendix 1). For timedependent simulation, we assumed $A_{0}=1$ and $m=0.1$ or 0.2 . Comprehensive numerical calculations were carried out for the initial values of the prey number, $x_{i n}=2$ and predator $y_{\text {in }}=2.5$ in a wide range of period $T$. Particular 


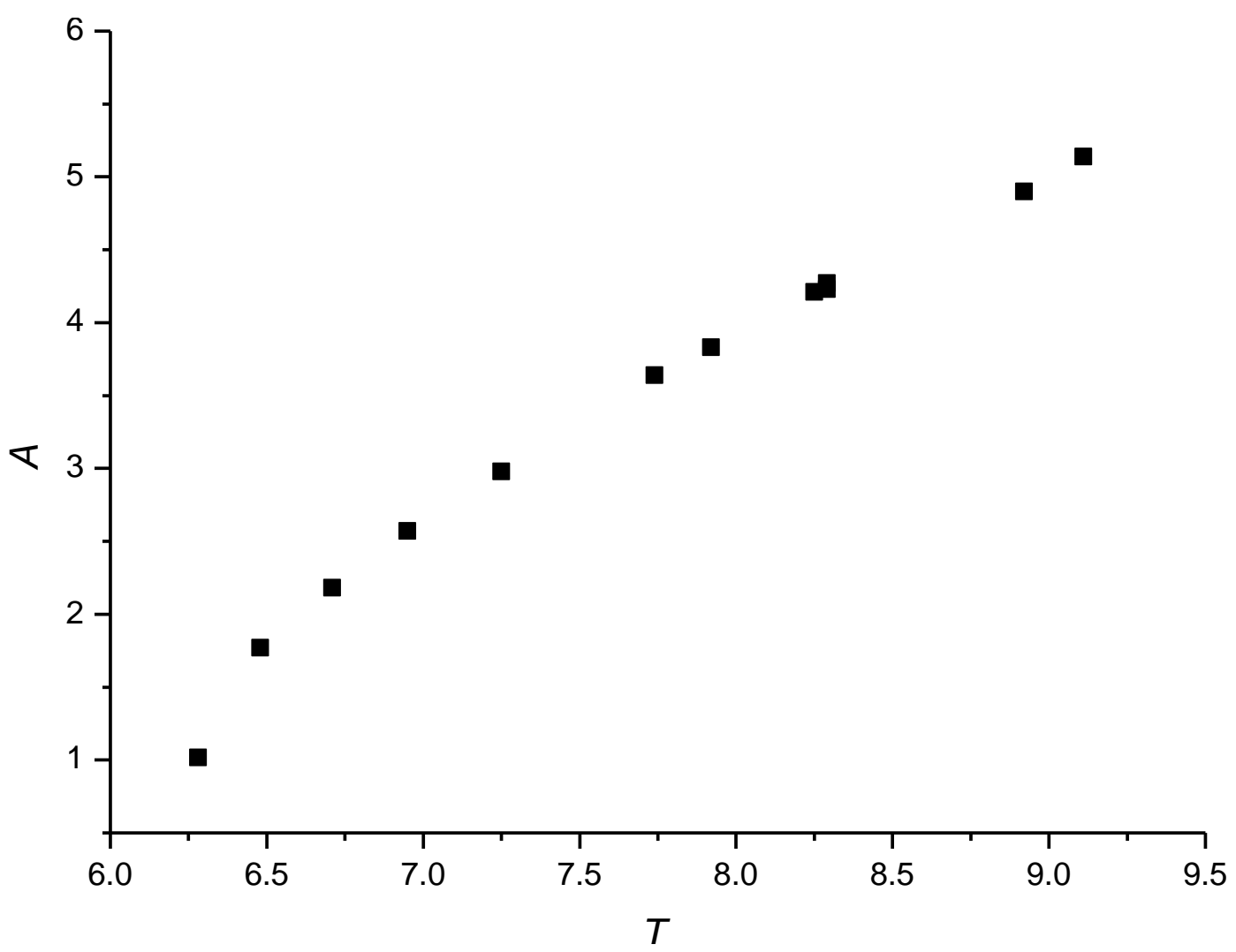

Figure 1. The period $T$ of natural oscillations of the system in dependence on its amplitude $A$ obtained by numerical calculations.

attention was paid to resonances, where in this nonlinear system the first and higher harmonics were expected to occur.

The range of oscillating, phase shift of $x \varphi_{x}$ can be associated with the resonance of the system stimulated by changes of environmental resources of food. Due to a strong nonlinearity of the system, resonance effects should be expected not only for the basic frequency (the first harmonic), but also for higher harmonics. Special simulation of behavior of the prey-predator system was carried out for the second harmonic case when the stimulated frequency was about two times higher than the natural frequency (Figure 2). Based on the numerical calculations, the amplitude of the number of prey $x$ and the phase shift $\varphi_{x}$ are very sensitive to the stimulated frequency $\omega$. The vibration amplitude of the system fluctuates first when it reaches a maximum value and drops next to a minimum of one (Figure 2a). This behavior can be explained by an increase in the fashion, the period of pendulum increases with its amplitude, which may be proved at a simple laboratory oscillation period of the system with increasing amplitude. This has a significant influence on the phase difference between vibrations of $A$-parameter and the system oscillations. When the two vibrations are in phase coincidence, the ampli- tudes of $x$ and $y$ components rise, and the period of the system oscillations gets longer, which leads to a delay of the vibration of the system in respect to the stimulated oscillations. After a while, these two oscilla-tions are not in phase, so the natural oscillation be-comes suppressed and its amplitude falls down consi-derably. Due to that, the frequency of the system osci-llations becomes larger than the stimulated oscillation, the phase delay disappears, and the oscillations again are in phase coincidence. Then the cycle of changes of the system amplitude and the phase relations are re-peated (Figure $2 b$ ). For other frequencies of stimulated vibrations which do not differ significantly and which are situated in the resonance range, the situation is similar. Only the resonance curves take different shapes, depending on frequency $\omega$. What is essential is that the frequency system gets adjusted to $\omega$. However, this ability to adjust is limited. When the phase difference becomes higher, the system cannot compensate for the frequency gap, and $\varphi_{x}$ decreases or increases monotonously. The maximum amplitude of the system drops suddenly to achieve approximately the initial value.

For example, Figure $2 b$ and $c$ present the time dependencies of $x$ and $\varphi_{x}$ for the periods $(T=2 \pi / \omega)$ equal to 3.36 and 3.3598 , respectively. It is normal that very small 

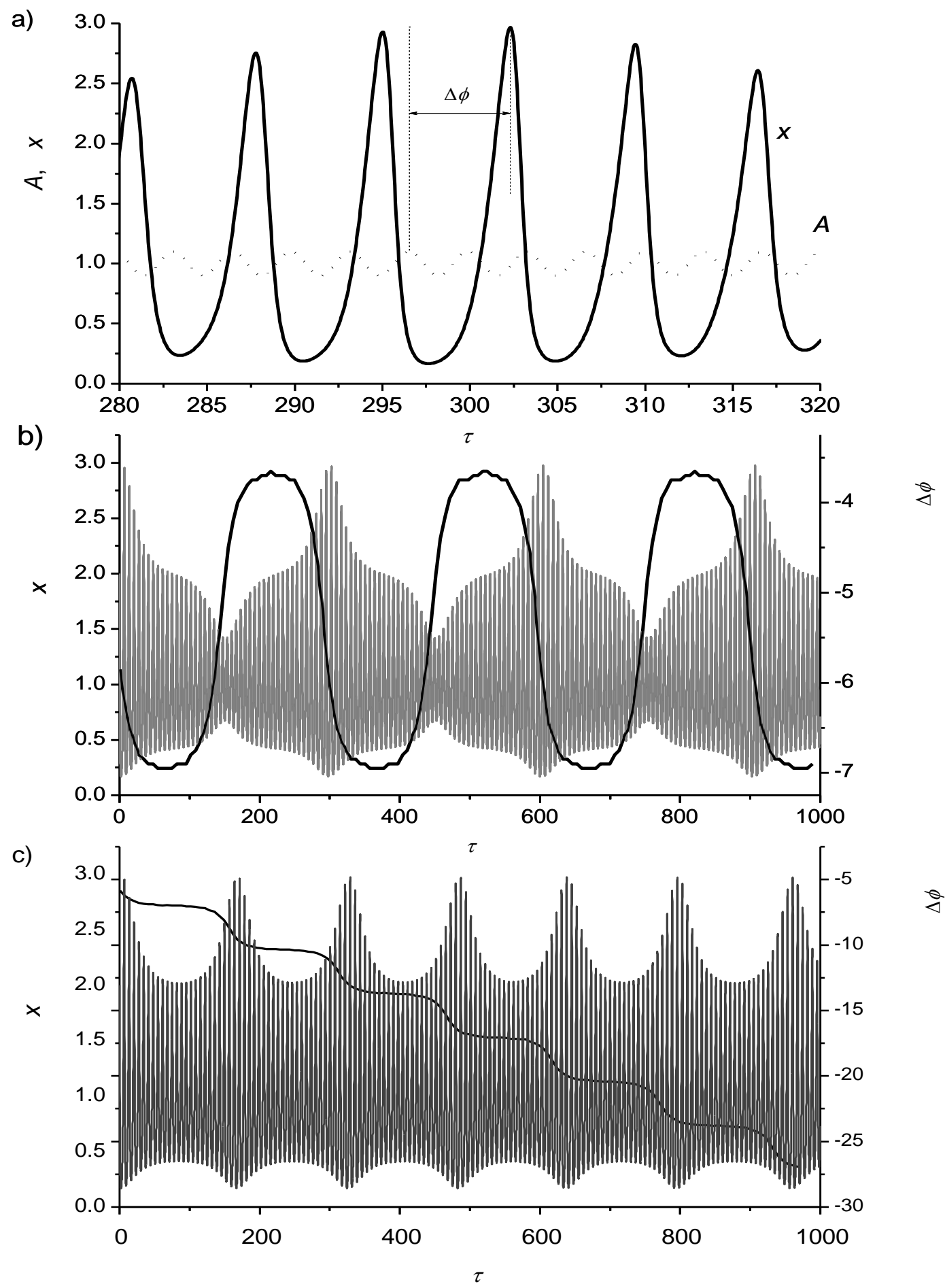

Figure 2. a) Changes of the population of preys $x$ stimulated by oscillatory changes of food $A$ consumed by preys. The phase shift $\Delta \varphi$ between the two oscillations is marked by arrow. b) and c) Population of the preys (the grey line) and the phase shift $\Delta \varphi$ (the solid line) for the period stimulated oscillation $T=3.36$ (b) and $T=3.3598$ (c).

changes of the stimulated frequency with a small ampitude $\left(m<A_{o}\right)$ cause sudden and significant changes in both the amplitude of prey (or predators) and phase shift $\varphi_{x}$. Furthermore, $\varphi_{x}$ initiated for the period 3.36 is maintained until 4.00 and its changes are less than $1 / 2$ of the natural oscillation of the system. Until $T=3.36$ the 
a)

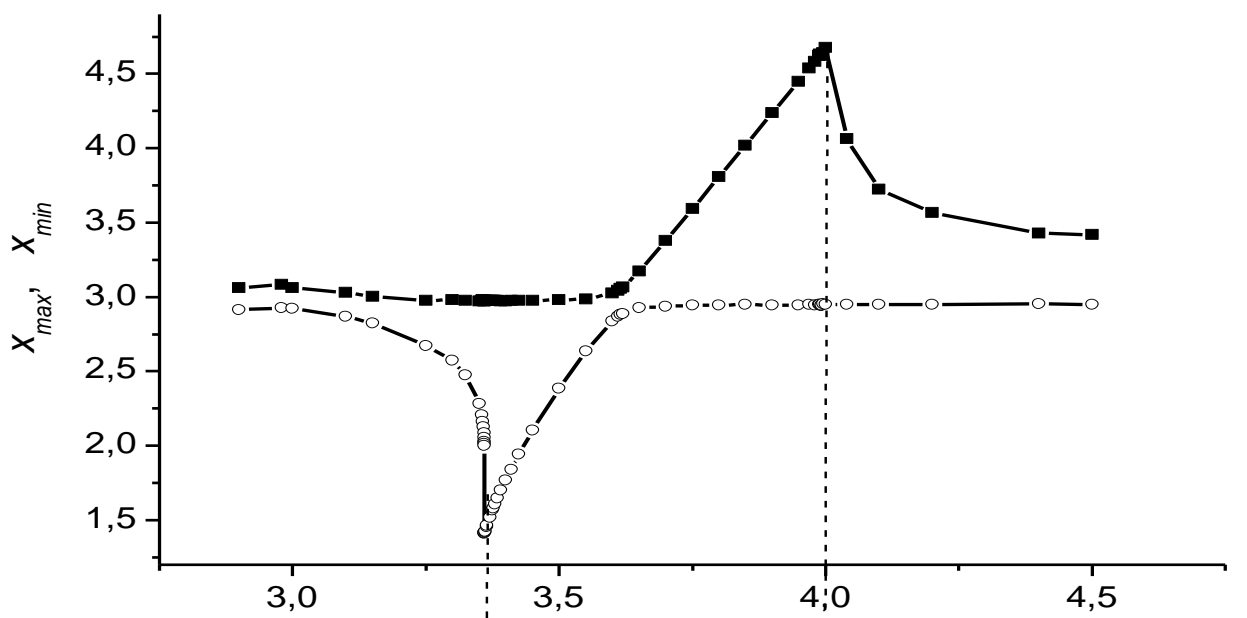

b)

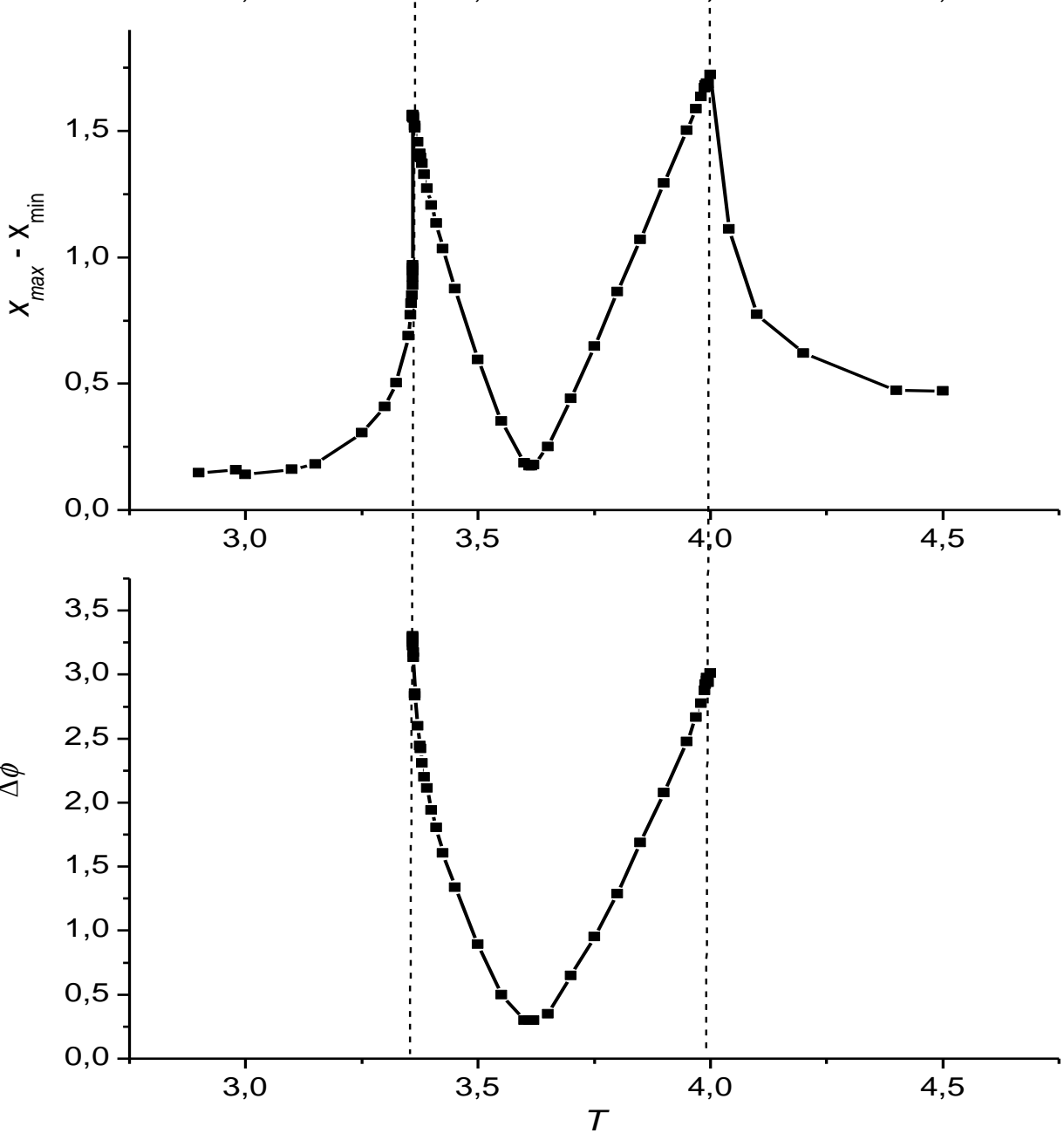

Figure 3. a) The maximum $x_{\max }$ and minimum $x_{\min }$ population number of preys and their difference b) in dependence on the period of the stimulated frequency for the second harmonic; $c$ ) the phase shift $\Delta \varphi$ between stimulated vibration and population of the preys in the resonance range.

phase shift decreases for decreasing $T$, first gradually in accordance with the amplitude oscillation of $x$, then the changes become smaller, and a decrease in $\varphi_{x}$ is monotonous. Above $T=4.00, \varphi_{x}$ increases, first gradually, then monotonously.

A response of Lotka-Volterra system to a second harmonic is shown in Figure 3. The amplitudes of $x$ at maximum, $x_{\max }$, and at minimum, $x_{\min }$ are determined from 


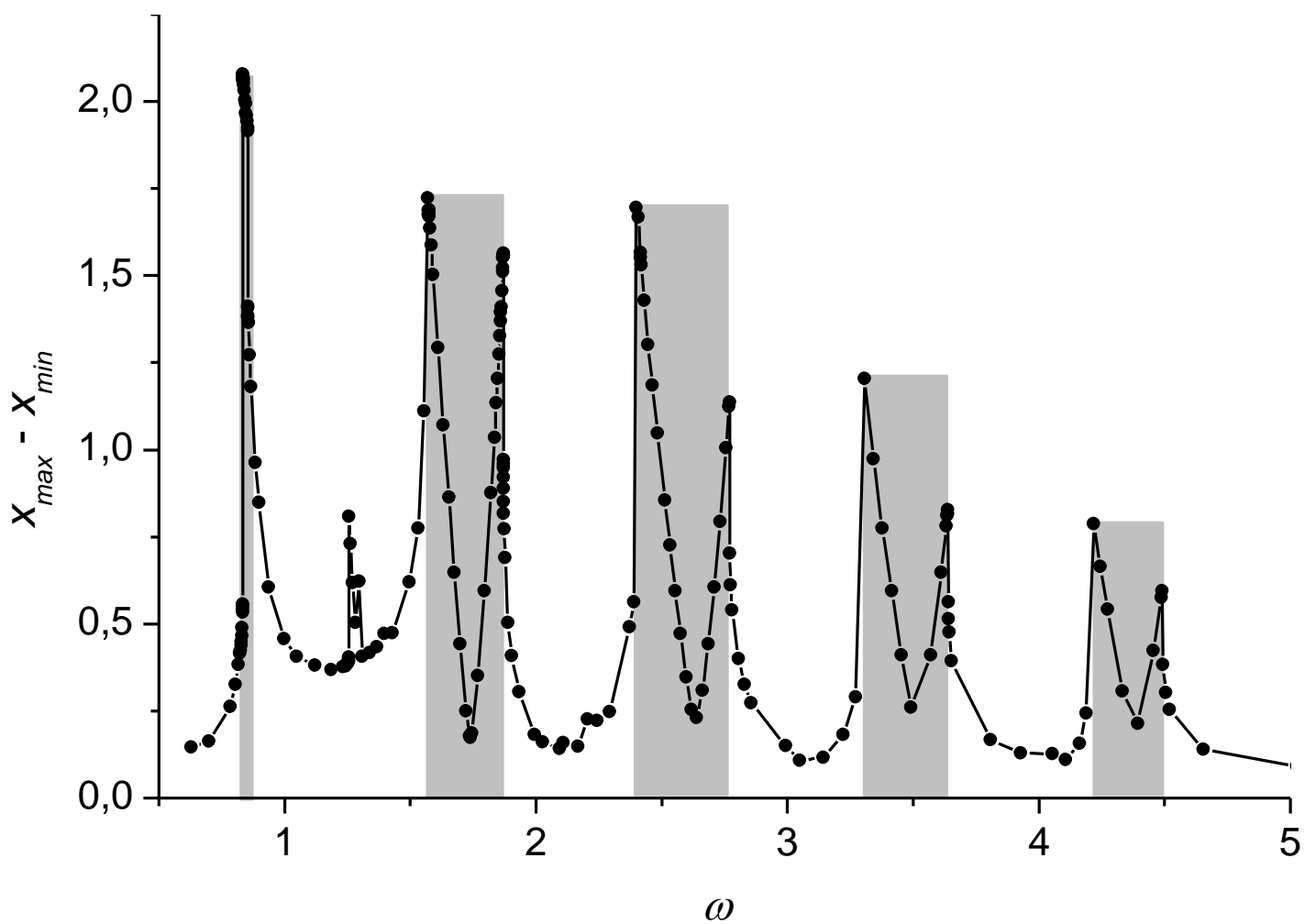

Figure 4. The change of prey population, $x_{\max }-x_{\min }$, in a function of stimulated frequency. The resonance ranges of orders from 1 to 5 are denoted by grey areas.

the phase trajectories, which are similar to the plots in Figure $2 \mathrm{~b}$. Abrupt changes can be noticed when the period $T$ of stimulated oscillation achieves the value, 3.36 or 4.00 (Figure 3a). The difference, $x_{\max }-x_{\min }$, is plotted in Figure $3 \mathrm{~b}$. Figure $3 \mathrm{c}$ shows the phase shift $\Delta \varphi$ is restricted only to the resonance range. It is normal that $x_{\max }-x_{\min }$ and $\Delta \varphi$ exhibit the minimum inside the resonance region.

A similar behavior was observed for other harmonics; however, for the first harmonic the range of the resonance is considerably lower and devoid of the minimum of the difference $x_{\max }-x_{\min }$ inside the resonance curve (Figure 4). This figure presents complex changes of population of prey, $x_{\max }-x_{\min }$, in function of the stimulation frequency $\omega$ (this dependence is similar to population of predators). Five resonances corresponding to five harmonics ( 1 to 5 ) are denoted by the shadowed regions. The resonance peak between the first and second harmonic for the stimulated frequency $\omega$ equal to $3 / 2$ of the frequency of the system is distinctly visible.

\section{Species extinction}

Predator - prey dynamics represented by Lotka-Volterra model (equations 1 or 2 ) is based on the following assumptions: (i) Predators eat only one kind of prey.

(ii) There is a particular case when the prey population considerably decreases and their population density becomes very low.

(iii) There is density of prey below when the population of predators decreases; this enables the recovery of the prey population because of lack of natural enemies.

Now, we shall discuss the prey-predator interaction introducing an additional assumption.

(iv) In the absence of predators the prey population considerably grows and tends towards a stable value determined by limited food resources. In this case, to the prey equation a logistic term proportional to $x^{2}: k x^{2}$ should be added, where $k$ is a constant.

$\frac{d x}{d \tau}=A x(1-y)-k x^{2}, \quad \frac{d y}{d \tau}=y(x-1)$.

Parameter $A$ periodically changed in time as described by eq. (3). The system (4) has asymptotically stable periodic solutions. Our simulation procedure is carried out for the first resonance harmonic of stimulated vibration for the period $T=7.8$. For $k=0.0003$, the unusually large changes in the prey and predator populations are reached. At the minimum, the prey and predator populations are a bit less than 0.028 and we may say that this value is sufficiently low to fulfil the assumption (iii). 

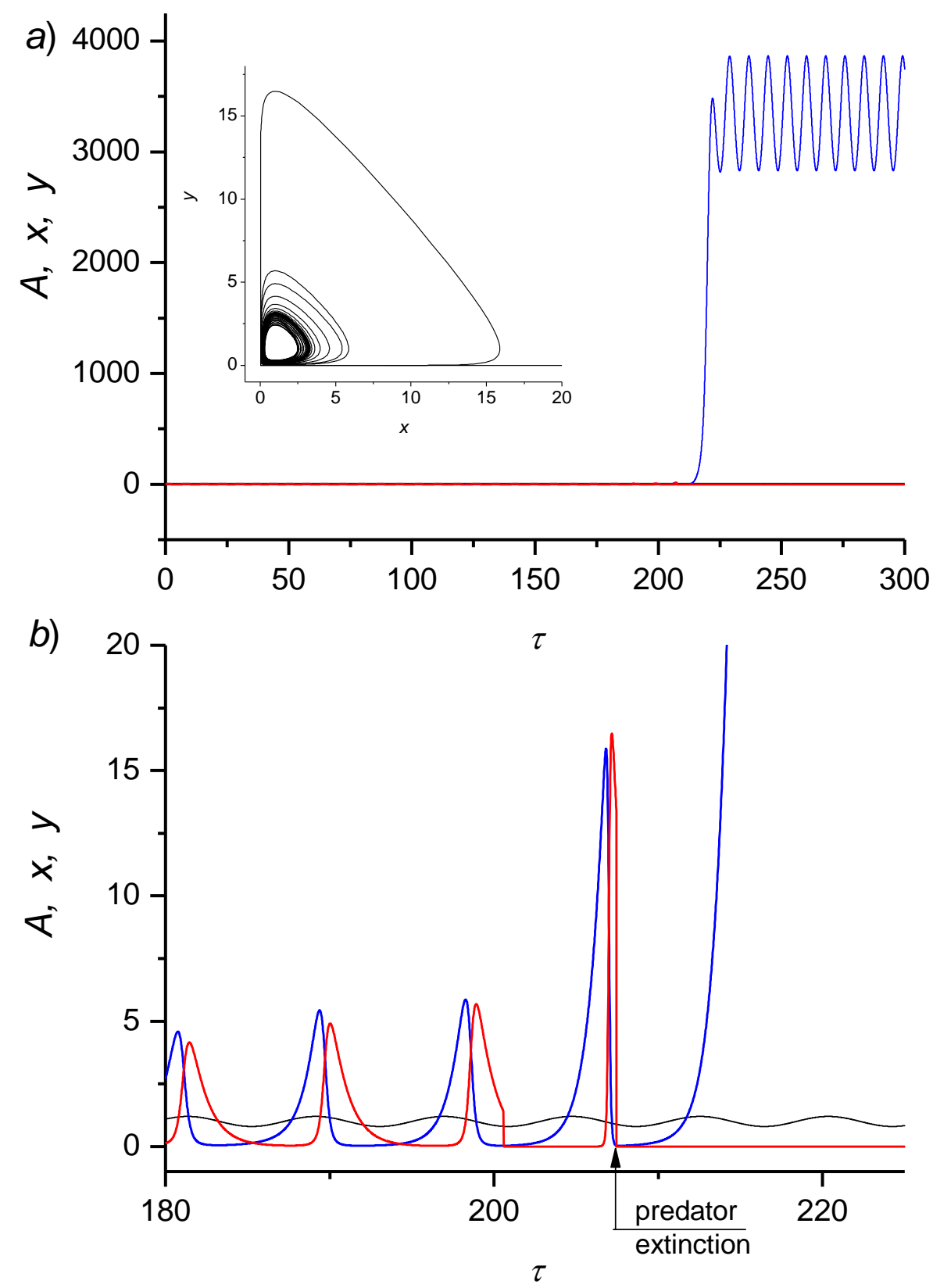

Figure 5. a) Changes in the food resources $A$ and populations of preys $x$ (the blue line) and predators $y$ (the red line) as function of time $T$ at the resonance (see text). Insert represents the phase trajectory. b) The same as in a) plotted in considerably expanded vertical axis. The simulation was made for the following data: $x_{i n}=2, y_{\text {in }}=2.5, T=7.8$, $A_{0}=1, m=0.2$, and the iteration step $h=T / 1000, k=0.0003$.

The predator population decays to zero; $y=0$ and $\frac{d y}{d \tau}=0$ in Equations (4). The result of the simulation is shown in Figure 5 applying the numerical procedure described in Appendix I. The prey population $x$ after extinction of predators considerably increases and then oscillates with respect to the stable value determined by the food resources represented by $A$. The values of $A$ and $y$ are significantly lower than $x$ and so they are not visible in
Figure $5 \mathrm{a}$ (the overlapping lines parallel to the abscissa axis). For this reason, in Figure $5 b$ the vertical axis is appropriately extended and the changes in $A, x$ and $y$ and their relations are visible. Insert in Figure 5a represents the phase trajectory, which tends to the triangular shape, which shows that the system is highly non-linear. It is clearly visible in the last cycle, when after a great increase in the species population it falls to a very small value and leads to extinction of predators and a sig- 
nificant growth of the population of prey.

\section{DISCUSSION}

The results of the present study illustrate only a small, but fundamental part of a more common phenomenon, which we should expect to occur if other parameters of the system, e.g. initial conditions or amplitude of the forced oscillations, were to change. The periodical inflows have been presented by many authors from different points of views (which were presented in introduction). In particular, the periodical modulation by pulse-shaped food inflow was considered too (Kneubühl, 1997). The results, in general, are similar to the above outcome for the sinusoidal food inflow, but they are different in details.

Forced oscillation seems to be important for characterizing the system. Response of a nonlinear oscillator to an external periodic force was sought for by Minorsky (1962) and Kneubühl (1997). For the system that is far from equilibrium, chaotic effects are also observed (Nicolis and Prigogine, 1977). Since the LotkaVolterra non-linear system is very sensitive, the initial conditions of the resonance effect depend on the conditions of the system. In contrast, for the linear oscillatory systems such effects as sensitivity to the initial conditions, resonance or chaotic behavior and triangular limit cycle are never observed.

In the linear dynamical systems the results may be utilized to construct the universal superposition principle, including the relaxation behavior. In the nonlinear dynamical systems the superposition principle does not apply. The facts presented above distinguish all nonlinear systems from the linear ones. The simple example of a linear oscillatory system is a damping harmonic oscillator (RLC circuit) whose amplitude varies slowly and periodically (Krupska and Krupski, 2003). When this linear oscillatory system (RLC circuit) is in resonance we can estimate a relaxation time from the phase shift between the generator voltage and the voltage of the capacitor of the RLC circuit. In the presented oscillatory nonlinear Lotka-Volterra system with the $A$ parameter periodically modulated, any relaxation occurs. This is non-relaxing system. In the forced periodically LotkaVolterra system higher harmonics are observed corresponding to the more than one resonance region. In the forced periodically RLC circuit higher harmonics are not observed, because only one resonance region occurs.

Kneubühl (1997), in his book, presents both linear and nonlinear physical and biological oscillatory systems, including Lotka-Volterra one. This book is a good comparison between the liner and nonlinear oscillatory systems. Another difference between the linear and nonlinear systems is visible in the shape of the resonance curve. The linear response, with a normal simple curve, Lorentzian or Gaussian in shape, can be observed only at the natural frequency of the system; while the non-linear linear shape of the resonance starts suddenly, then it is kept within a certain frequency range and finishes also suddenly. The higher harmonics of the resonance frequency are observed, which spread over a significant range of the frequency. Sometimes, in the resonance region different modes may be observed, depending on the initial conditions of the system and on the amplitude modulation of the forced oscillations. These facts lead to the finding of a lot of other forced frequency (or periods) for which the oscillation of the prey-predator system becomes large and leads to extinction of species, as in the example shown in Figure 5. That is why LotkaVolterra system is interesting and requires further investigations.

The predator extinction follows after a lot of oscillations of the system (for example 27 periods in Figure 5). It explains the ecological behaviour of the predator-prey system, in which after a long time of the predator-prey coexistence, significant growth of the system oscillations occurs. The predator population decreases below critical value and again its growth becomes impossible. This situation results from lack of food for predators, because of significant decrease of prey and their dispersion in the environment as well as difficulties of the predators to reproduce. The preys are in better situations because they have sufficient food resources and their population growth is approximately logistical and finally oscillate according to the oscillation of the food in the environment.

In this paper, a typical two-dimensional nonlinear system is considered. In this system there is no effect of chaos. It is a stable system, where chaos does not occur. There is no damping oscillation here. In the twodimensional non-linear systems, chaos and stochastic fluctuations usually do not occur. As a result, stochastic fluctuations are neglected in the model presented in this paper. We may suppose that in our model these fluctuations do not occur or are so small that they can be neglected. In the literature three or four-dimensional examples of a competitive Lotka-Volterra system have been characterized as where stochastic fluctuations occur (Vano, 2006; Sprott, 2005). There is no work on the two-dimensional nonlinear regarding Lotka-Volterra model and other systems in which there are stochastic processes. In the stochastic or random process there is some indeterminacy. This means if the initial condition is known, there are several directions in which the process may evolve. In our case at given initial condition there is only one process of evolution of system. So this is not a stochastic process. But, we observe a strong sensitivity to initial conditions (Figure 2).

The most interesting conclusion which we may draw for the oscillatory forced prey-predator system described above is the significant increase in the amplitude of the populations and possibility ofextinction of predators during time, considerable larger than the oscillation period of the system. Therefore, the above presented modification of Lotka-Volterra model may be used to ex- 
plain some examples of species extinction. Although, we should note that Volterra-Lotka type of model (3) is a conservative system without dissipation. We should note also that the model (4) is not generic for $k=0$, meaning that infinite small change of this parameter leads to a qualitative different solution.

In this paper, an ideal example of stimulated oscillation with constant amplitude is considered, but in the real systems, we have very irregular changes in the environmental conditions driven by noise and some other periodical parameters. For simplicity, we assumed only one alternating parameter $(A)$, which leads to the effect of the species extinction, using the simple numerical calculations. This may be the weakness of the presented model, but this is strictly a simulation paper. It is not possible to simultaneously simulate other time-varying parameters that describe Lotka-Volterra system. We chose only one modulation parameter $A$, which seems to be the most important. We hope the model presented in this paper may be useful for students.

\section{ACKNOWLEDGEMENTS}

I would like to thank Prof. IFMPAN M. Krupski for being helpful in the discussion during the preparation of this paper.

\section{REFERENCES}

Ackland GJ, Gallagher ID (2004). Stabilization of large generalized Lotka-Volterra foodwebs by evolutionary feedback. Phys. Rev. Letters 93(15):158701-1-4.

Arditi R, Ginzburg LR (1989). Coupling in predator-prey dynamics: ratiodependence. J. Theoretic. Biol. 139:311-326.

Arinstein A, Gitterman M (2008.) Inverting spring pendulum driven by a periodic force: linear versus nonlinear analysis. Eur. J. Phys. 29:385392.

Coppex F, Droz M, Lipowski A (2004). Extinction dynamics of LotkaVolterra ecosystems on evolving networks. Phys. Rev. E. 69:0619011-6.

Cressman R, Garay J (2003). Evolutionary stability in Lotka-Volterra systems. J. Theoretic. Biol. 222:233-245.

Dutta D, Bhattacharjee JK (2008). Lotka-Volterra dynamics under periodic influence. Nonlinear Sciences. Chaotic Dynamics. arXiv:nlin/0610044v1.
Gilchrist MA, Coombs D (2006). Evolution of virulence: interdependence, constraints and selection using nested models. Theoretical. Pop. Biol. 69:145-153.

Kahn PB, Zarmi Y (2003). Weakly nonlinear oscillations: a perturbative approach. Am. J. Phys. 72(4):538-552.

Khaminskii RZ, Klebaner FC, Liptser R (2003). Some results on the Lotka-Volterra model and its small random perturbations. Acta Applicandae Mathematicae. 78:201-206.

Kneubühl FK (1997). Oscillations and waves. Springer-Verlag Berlin Heidelberg, chapter 3.

Krupska A, Krupski M (2003). Study of the phase delay in the amplitude-modulated harmonic oscillator. Eur. J. Phys. 24:429-433.

Liu B, Chen L (2004). The periodic competing Lotka-Volterra model with impulsive effect. Mathematical Medicine and Biology. 21(2):129-145.

Lotka AJ (1925). Elements of Physical Biology. Williams and Wilkins, Baltimore, MD.

MacArthur RH, Connell JH (1966). The biology of populations. John Wiley \& Sons, New York.

Minorsky N (1962). Nonlinear oscillations. Van Nostrand, Princeton. N.J.

Morozov Ayu, Adamson MW (2011). Evolution of virulence driven by predator-prey interaction: possible consequences for population dynamics. J. Theoretic. Biol. 276:181-191.

Nicolis G, Prigogine I (1977). Self-Organization in Nonequilibrium Systems. Wiley-Interscience, New York.

Rosenzweig MP, MacArthur RH (1969). Graphic representation and stability conditions of predator-prey interaction. Am. Nat. 97:209-223.

Smith M (1978) Models in Ecology. Cambridge University Press, Cambridge.

Sprott JC, Wildenberg JC, Azizi Y (2005). A simple spatiotemporal chaotic Lotka-Volterra model. Chaos, Solitons and Fractals 26:10351043.

Svennungsen TO, Kisdi E (2009). Evolutionary branching of virulence in a single-infection model. J. Theoretic. Biol. 257: 408-418.

Turchin P (2003). Complex population dynamics: a theoretical/empirical synthesis. Princeton University Press.

Vano JA, Wildenberg JC, Anderson MB, Noel JK, Sprott JC (2006). Chaos in Low-Dimensional Lotka-Volterra Models of Competition. Nonlinearity.19:2391-2404.

Volterra V (1926). Sulle variazioni e fluttuazioni di specie animali conviventi. Memorie della R. Aceademia dei Lincei S, 6(2):31-113. 


\section{Appendix I}

LV program;

\{Lotka-Volterra Model\}

Constant:

$\mathrm{t} 0=0$;

$\mathrm{x} 0=2$

$\mathrm{y} 0=2.5$

Ao $=1$

$\mathrm{m}=0.2$;

$\mathrm{Tz}=7.8$;

$\mathrm{h}=\mathrm{Tz} / 1000$;

$\mathrm{k}=0.0003$;

var

$\mathrm{t}, \mathrm{x}, \mathrm{y}, \mathrm{x} 1, \mathrm{y} 1, \mathrm{~K}$ : Real;

$\mathrm{n}$ : Longlnt;

k1, k2, k3, k4 : array[1..2] of Real;

plik1 : text;

function fna(x, y, t: Real): Real;

begin

fna := $A^{*}\left(x-x^{*} y\right)-k^{*} x^{*} x$;

end;

function $\mathrm{fnb}(\mathrm{x}, \mathrm{y}$, t: Real): Real;

begin

$f n b:=y^{*}(x-1)$;

end;

begin

Assign (plik1,'C:ILV.dat');

Rewrite (plik1);

$x:=x 0 ; y:=y 0$ for $\mathrm{n}:=0$ to 10000 do

begin

$\mathrm{t}:=\mathrm{t} 0+\mathrm{n}^{\star} \mathrm{h} ; \mathrm{x}:=\mathrm{x}+\mathrm{x} 1 ; \mathrm{y}:=\mathrm{y}+\mathrm{y} 1$

$k 1[1]:=f n a(x, y, t)^{*} h ;$

$\mathrm{k} 1[2]:=\mathrm{fnb}(\mathrm{x}, \mathrm{y}, \mathrm{t})^{*} \mathrm{~h}$;

$k 2[1]:=f n a(x+k 1[1] / 2, y+k 1[2] / 2, t+h / 2)^{*} h$;

$k 2[2]:=f n b(x+k 1[1] / 2, y+k 1[2] / 2, t+h / 2)^{*} h$;

$k 3[1]:=f n a(x+k 2[1] / 2, y+k 2[2] / 2, t+h / 2)^{*} h$;

$k 3[2]:=f n b(x+k 2[1] / 2, y+k 2[2] / 2, t+h / 2)^{*} h$;

$k 4[1]:=f n a(x+k 3[1], y+k 3[2], t+h)^{*} h$;

$k 4[2]:=f n b(x+k 3[1], y+k 3[2], t+h)^{\star} h$;

$x 1:=\left(k 1[1]+2^{*} k 2[1]+2^{*} k 3[1]+k 4[1]\right) / 6$;

$y 1:=\left(k 1[2]+2^{*} k 2[2]+2^{*} k 3[2]+k 4[2]\right) / 6$;

$A:=A o^{*}\left(1+m^{*} \operatorname{Sin}\left(2^{*} \mathrm{Pi} / T z^{*} \mathrm{t}\right)\right)$;

IF $x<=0.028$ THEN $y:=0$;

Writeln(plik1, t, ' ', A,' ', x,' ',y);

end;

close(plik1);

end. 